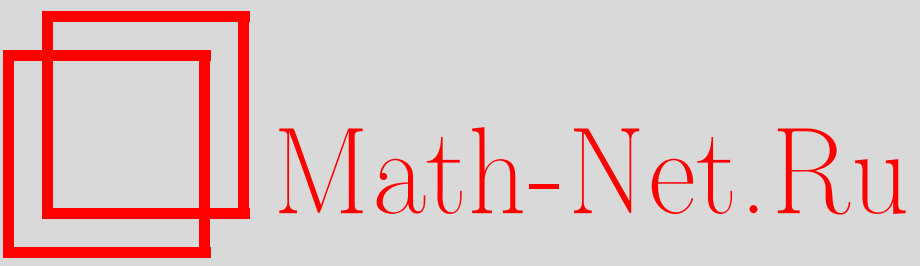

N. Chevallier, Law of the sum of Bernoulli random variables, Теория вероятн. и ее примен., 2010, том 55, выпуск 1, 87-103

DOI: https://doi.org/10.4213/tvp4177

Использование Общероссийского математического портала Math-Net.Ru подразумевает, что вы прочитали и согласны с пользовательским соглашением

http://www.mathnet.ru/rus/agreement

Параметры загрузки:

IP : 54.80 .73 .141

26 апреля 2023 г., 17:07:28 


\title{
LAW OF THE SUM OF BERNOULLI RANDOM VARIABLES
}

\begin{abstract}
Пусть $\Delta_{n}$ - множество всех возможных совместных распределений $n$ бернуллиевских случайных величин $X_{1}, \ldots, X_{n}$. Предположим, что $\Delta_{n}$, являющееся симплексом в $2^{n}$-мерном пространстве, снабжено нормированной мерой Лебега $\mu_{n}$. Предположим также, что целое число $n$ велико. Мы показываем, что тогда существует подмножество $\Delta$ множества $\Delta_{n}$, мера $\mu_{n}(\Delta)$ которого очень близка к единице, такое, что если совместное распределение величин $X_{1}, \ldots, X_{n}$ принадлежит $\Delta$, то закон распределения суммы $X_{1}+\cdots+X_{n}$ близок к биномиальному закону $\mathscr{B}(n, 1 / 2)$. Этот результат не опирается ни на какое предположение независимости. Кроме того, мы доказываем результат подобного рода в случае, когда на $\Delta_{n}$ задана другая вероятностная мера $\nu_{n}$.
\end{abstract}

Ключевые слова и фразы: бернуллиевская случайная величина, биномиальный закон.

1. Introduction. The most common explanation for the ubiquity of the Gaussian law is the Central Limit Theorem. Another explanation closely related to the previous one, is that the Gaussian law is the only stable law with finite variance. The proofs of the Central Limit Theorem always rest on some independence assumption or at least on some stationarity assumption. The purpose of our work is to give in a very simplified situation, another kind of explanation for the ubiquity of the Gaussian law.

Consider a sequence $X_{1}, \ldots, X_{n}$ of Bernoulli random variables. We are interested in the law of the sum $S_{n}=X_{1}+\cdots+X_{n}$ without any independence assumption about the random variables $X_{i}$. Since the Laplace-Moivre theorem asserts that up to a suitable normalization, for $n$ large (and $p$ not too small), the binomial distribution $\mathscr{B}(n, p)$ is close to the Gaussian law, an explanation for the ubiquity of the Gaussian law may be in our setting: when $n$ is large, the law of $S_{n}$ is often very close to the symmetric binomial distribution $\mathscr{B}(n, 1 / 2)$.

We must explain what we mean by often. Fix a positive integer $n$. Denote by $\Delta_{n}$ the set of all possible joint distributions of $n$ Bernoulli random variables $X_{1}, \ldots, X_{n}$. Then $\Delta_{n}$ is the set of all probability measures on

*Université de Haute Alsace, 4, rue des frères Lumière, 68093 Mulhouse, France; e-mail: nicolas.chevallier@uha.fr 
$\{0,1\}^{n}$. To each element $p=\left(p_{i}\right)_{i \in\{0,1\}^{n}}$ of $\Delta_{n}$, one can associate the law of the sum $S_{n}=X_{1}+\cdots+X_{n}$. It is a probability measure $L_{n}(p)$ on the set $\{0, \ldots, n\}$. When we choose the uniform probability law $b=\left(2^{-n}, \ldots, 2^{-n}\right)$ on $\{0,1\}^{n}$, we get $L_{n}(b)=\mathscr{B}_{n}=\mathscr{B}(n, 1 / 2)$, the symmetric binomial distribution:

$$
\mathscr{B}_{n}(k)=C_{n}^{k} \cdot 2^{-n}
$$

for all $k$ in $\{0, \ldots, n\}$. Let $\varepsilon>0$, our aim is to estimate the size of the set $\Delta_{n, \varepsilon}$ of probability measures $p$ in $\Delta_{n}$ such that for all $k$ in $\{0, \ldots, n\}$,

$$
\left|L_{n}(p)(k)-\mathscr{B}_{n}(k)\right| \leqslant \varepsilon .
$$

To make precise this question, we have to measure the size of subsets of $\Delta_{n}$. This can be done with the help of a probability measure on $\Delta_{n}$. If $\mu$ is a probability measure on $\Delta_{n}$, we wish to prove that

$$
\mu\left(\Delta_{n, \varepsilon}\right)
$$

is close to 1 when $n$ is large. In a less formal language: when $n$ is large, choosing at random the joint distribution of $\left(X_{1}, \ldots, X_{n}\right)$, it is likely that the law of $S_{n}$ is very close to the symmetric binomial distribution $\mathscr{B}(n, 1 / 2)$.

2. Statements of results. There are many choices for the probability measure $\mu$ and we shall only consider two. The first and the most natural one is $\mu=\mu_{1, n}$, the normalized Lebesgue measure on $\Delta_{n}$. Our first result is the following theorem.

Theorem 1. There exists a constant $A$ such that for all positive integers $n$ and all positive numbers $\varepsilon$,

$$
\begin{aligned}
& \mu_{1, n}\left(\Delta_{n, \varepsilon}\right) \geqslant 1-\frac{A \sqrt{n}}{\varepsilon^{2} \cdot 2^{n-1}}, \\
& \mu_{1, n}\left(\left\{p \in \Delta_{n}: \sup _{I \subset\{0, \ldots, n\}}\left|L_{n}(p)(I)-\mathscr{B}_{n}(I)\right| \leqslant \varepsilon\right\}\right) \geqslant 1-\frac{A n^{5 / 2}}{\varepsilon^{2} \cdot 2^{n-1}} .
\end{aligned}
$$

Though the Lebesgue measure is very natural, it has a drawback. One can consider the law of the first $n-1$ Bernoulli random variables $\left(X_{1}, \ldots, X_{n-1}\right)$ as random variable defined on $\Delta_{n}$. This random variable is the projection

$$
\begin{aligned}
\operatorname{pro}_{n}: & \Delta_{n} \rightarrow \Delta_{n-1} \\
: & \left(p_{i}\right)_{i \in\{0,1\}^{n}} \mapsto\left(p_{(j, 0)}+p_{(j, 1)}\right)_{j \in\{0,1\}^{n-1}} .
\end{aligned}
$$

The point is that the Lebesgue measure $\mu_{1, n-1}$ is not the image of $\mu_{1, n}$ by the map $\operatorname{pro}_{n}$; the family $\left(\mu_{1, n}\right)_{n \geqslant 1}$ is not a projective family of probability measures. We would like to find a projective family $\left(\mu_{2, n}\right)_{n \geqslant 1}$ of 
natural probability measures on the sequence of sets $\left(\Delta_{n}\right)_{n \geqslant 1}$. This can be done inductively: when we know the law $\left(p_{i}\right)_{i \in\{0,1\}^{n}}$ of $\left(X_{1}, \ldots, X_{n}\right)$ the law $\left(p_{(i, j)}^{\prime}\right)_{i \in\{0,1\}^{n}, j \in\{0,1\}}$ of $\left(X_{1}, \ldots, X_{n}, X_{n+1}\right)$ is chosen at random uniformly among all the possible laws. Let us make it precise. Consider the natural map which is almost a bijection,

$$
\begin{aligned}
\psi_{n}: & \Delta_{n} \times[0,1]^{\{0,1\}^{n}} \rightarrow \Delta_{n+1} \\
: & \left(\left(p_{i}\right)_{i \in\{0,1\}^{n}},\left(x_{i}\right)_{i \in\{0,1\}^{n}}\right) \mapsto\left(p_{(i, j)}^{\prime}\right)_{i \in\{0,1\}^{n}, j \in\{0,1\}},
\end{aligned}
$$

where $p_{(i, 0)}^{\prime}=p_{i} x_{i}$ and $p_{(i, 1)}^{\prime}=p_{i}\left(1-x_{i}\right)$. The uniformity means that the choice of $\left(p_{(i, j)}^{\prime}\right)_{i \in\{0,1\}^{n}, j \in\{0,1\}}$ given $\left(p_{i}\right)_{i \in\{0,1\}^{n}}$ is done at random with respect to the Lebesgue measure $\lambda_{n}$ on $[0,1]^{\{0,1\}^{n}}$. This enables one to transfer a probability measure from $\Delta_{n}$ to $\Delta_{n+1}$. If we have a probability measure $\mu_{2, n}$ on $\Delta_{n}$, the product of this measure with the Lebesgue measure $\lambda_{n}$ on $[0,1]^{\{0,1\}^{n}}$ gives rise to a measure on $\Delta_{n} \times[0,1]^{\{0,1\}^{n}}$ and its image by $\psi_{n}$ is a new measure $\mu_{2, n+1}$ on $\Delta_{n+1}$. Since the map $\operatorname{pro}_{n+1} \circ \psi_{n}: \Delta_{n} \times[0,1]^{\{0,1\}^{n}} \rightarrow$ $\Delta_{n}$ is the projection on $\Delta_{n}$, the image by $\operatorname{pro}_{n+1}$ of $\mu_{2, n+1}$ is $\mu_{2, n}$. Taking $\mu_{2,1}$ the normalized Lebesgue measure on $\Delta_{1}$, we get a sequence $\left(\mu_{2, n}\right)_{n \geqslant 1}$ of natural probability measures on the sequence of simplices $\left(\Delta_{n}\right)_{n \geqslant 1}$ (see Section 5 , for a purely probabilistic point of view about the measures $\mu_{2, n}$ ). This is our second choice which also has a drawback, since it looses the symmetry between the random variables

$$
\begin{aligned}
\operatorname{pr}_{i}: & \Delta_{n} \rightarrow \mathbf{R} \\
: & \left(p_{j}\right)_{j \in\{0,1\}^{n}} \mapsto p_{i},
\end{aligned}
$$

$i \in\{0,1\}^{n}$, as well as the symmetry between the variables $X_{i}, i \in\{1, \ldots, n\}$. Our second result is the following theorem.

Theorem 2. There exists a constant $C$ such that for all positive integers $n$ and all positive numbers $\varepsilon$,

$$
\begin{aligned}
& \mu_{2, n}\left(\Delta_{n, \varepsilon}\right) \geqslant 1-\frac{C \ln n}{n^{3 / 2} \varepsilon^{2}}, \\
& \mu_{2, n}\left(\left\{p \in \Delta_{n}: \sup _{I \subset\{0, \ldots, n\}}\left|L_{n}(p)(I)-\mathscr{B}_{n}(I)\right| \leqslant \varepsilon\right\}\right) \geqslant 1-\frac{C \ln ^{3 / 2} n}{\sqrt{n} \varepsilon^{2}} .
\end{aligned}
$$

$\mathrm{R} \mathrm{e} \mathrm{m}$ a $\mathrm{r} \mathrm{k}$. In both Theorems 1 and 2 it is possible to find an explicit value for the constants $A$ and $C$. It is easy to check that the value $A=2$ works in Theorem 1 whereas it is more difficult to give an explicit value for the constant $C$ and we do not give any.

The main point of these two results is that they do not need any independence assumption about the variables $X_{i}$. There must be some other works of the same kind but we have only find one: K. Takeuchi and A. Takemura [4] have studied the law of the sum $S_{n}=X_{1}+\cdots+X_{n}$, where the $X_{i}$ are 
Bernoulli variables. They only assume some condition about «central binomial moments» which are one-to-one functions of the factorial moments (the $k$-th factorial moment of random variable $X$ is $\mathbf{E}[X(X-1) \cdots(X-k+1)])$. This allows them to prove convergence to the normal law or to the Poisson law for a triangular array of Bernoulli variables $X_{i, n}$. Their hypotheses are only about the central binomial moments of $S_{n}=X_{1, n}+\cdots+X_{n, n}$.

3. Sketch of proofs. The ideas of the proofs of Theorems 1 and 2 are exactly the same. It is the reason why, although these proofs are not difficult, we begin by describing their main steps. For $i=1,2, n \in\{1,2, \ldots\}$ and $k \in\{0, \ldots, n\}$, denote by $\mathbf{E}_{i, n}(k)$ and $\mathbf{V}_{i, n}(k)$ the expectation and the variance of the random variable $p \mapsto L_{n}(p)(k)$ defined on the probability space $\left(\Delta_{n}, \mu_{i, n}\right)$.

Making use of the symmetries, we replace the simplex $\Delta_{n}$ by another geometrical space, where the computation of expectations is easier. Then we show in both cases that

$$
\mathbf{E}_{i, n}(k)=C_{n}^{k} \cdot 2^{-n}=\mathscr{B}_{n}(k) .
$$

Since, by the Chebyshev inequality,

$$
\mu_{i, n}\left(\left\{p \in \Delta_{n}:\left|L_{n}(p)(k)-\mathbf{E}_{i, n}(k)\right|>\varepsilon\right\}\right) \leqslant \frac{\mathbf{V}_{i, n}(k)}{\varepsilon^{2}},
$$

Theorems 1 and 2 can be deduced from appropriate upper bound on the variances $\mathbf{V}_{i, n}(k)$. In the first case, standard results lead to the inequality

$$
\mathbf{V}_{1, n}(k) \leqslant \frac{C_{n}^{k}}{2^{2 n}}
$$

In the second case, computations are not as easy as in the first case. Some well-known estimates of binomial coefficients enable one to show that for all $k$ in $\{0, \ldots, n\}$,

$$
\mathbf{V}_{2, n}(k) \leqslant \frac{C}{n^{2}}
$$

and that for all $k$ such that $|k-n / 2| \geqslant 2 \sqrt{n \ln n}$,

$$
\mathbf{V}_{2, n}(k) \leqslant \frac{C}{n^{5}}
$$

where $C$ is a constant which does not depend of $n$ and $k$.

4. Proof of Theorem 1. 1. The cardinal number of the set $\Delta_{n}$ is $N=2^{n}$ and there is a one-to-one correspondence between the sets $\{0,1\}^{n}$ and $\{0, \ldots, N-1\}$. Therefore each $p$ in $\Delta_{n}$ can be seen as a probability measure on $\{0,1, \ldots, N-1\}$ :

$\Delta_{n}=\left\{\left(p_{0}, \ldots, p_{N-1}\right) \in \mathbf{R}^{N}: p_{0}, p_{1}, \ldots, p_{N-1} \geqslant 0, p_{0}+p_{1}+\cdots+p_{N-1}=1\right\}$. 
Furthermore, for all $k$ in $\{0, \ldots, n\}$, there is a subset $E_{k}$ of $\{0, \ldots, N-1\}$ with $C_{n}^{k}$ elements such that for all $p=\left(p_{0}, \ldots, p_{N-1}\right)$ in $\Delta_{n}$,

$$
L_{n}(p)(k)=\sum_{i \in E_{k}} p_{i}
$$

2. Let $\sigma$ be a permutation of the set $\{0, \ldots, N-1\}$. Then $\sigma$ induces the linear map, $f_{\sigma}\left(x_{1}, \ldots, x_{n}\right)=\left(x_{\sigma(0)}, x_{\sigma(2)}, \ldots, x_{\sigma(N-1)}\right)$, which sends $\Delta_{n}$ onto itself. Therefore the measure $\mu_{1, n}$ is $f_{\sigma}$-invariant. It follows that given a subset $E$ of $\{0, \ldots, N-1\}$, the distribution function of the map

$$
\Delta_{n} \ni p=\left(p_{0}, \ldots, p_{N-1}\right) \mapsto L_{E}(p)=\sum_{i \in E} p_{i}
$$

depends only on the cardinal number of $E$. This means that for all $k \in$ $\{0, \ldots, n\}$, the map $L_{E_{k}}$ has the same distribution as the map $L_{F_{k}}$, where

$$
F_{k}=\left\{0, \ldots, C_{n}^{k}-1\right\}
$$

Hence,

$$
\begin{aligned}
& \mu_{1, n}\left(\left\{p \in \Delta_{n}:\left|L_{n}(p)(k)-\mathscr{B}_{n}(k)\right| \geqslant \varepsilon\right\}\right) \\
& \quad=\mu_{1, n}\left(\left\{p \in \Delta_{n}:\left|L_{F_{k}}(p)-\mathscr{B}_{n}(k)\right| \geqslant \varepsilon\right\}\right) .
\end{aligned}
$$

3. In order to estimate $\mu_{1, n}\left(\left\{p \in \Delta_{n}:\left|L_{F_{k}}(p)-\mathscr{B}_{n}(k)\right| \geqslant \varepsilon\right\}\right)$, let us introduce another way to see the probability space $\left(\Delta_{n}, \mu_{1, n}\right)$. Let $\left(Y_{1}, \ldots, Y_{N-1}\right)$ be $N-1$ independent random variables uniformly distributed in the interval $[0,1]$. Arranging them in ascending order we find $N-1$ random variables $Z_{1} \leqslant \cdots \leqslant Z_{N-1}$. The joint distribution of $\left(Z_{1}, \ldots, Z_{N-1}\right)$ is the normalized Lebesgue measure $\nu$ on

$$
T=\left\{\left(z_{1}, \ldots, z_{N-1}\right) \in \mathbf{R}^{N-1}: 0 \leqslant z_{1} \leqslant \cdots \leqslant z_{N-1} \leqslant 1\right\} .
$$

Let $\varphi: \mathbf{R}^{N-1} \rightarrow \mathbf{R}^{N}$ be the map defined by

$$
\varphi\left(z_{1}, \ldots, z_{N-1}\right)=\left(z_{1}, z_{2}-z_{1}, \ldots, z_{N-1}-z_{N-2}, 1-z_{N-1}\right) .
$$

The image of $T$ by $\varphi$ is $\Delta_{n}$ and since the map $\varphi$ is affine, the image of the measure $\nu$ by $\varphi$ is the measure $\mu_{1, n}$. Now, let $F=\{0, \ldots, m-1\}$ be a subset of $\{0, \ldots, N-1\}$. For all $z=\left(z_{1}, \ldots, z_{N-1}\right) \in T$, we have $L_{F}(\varphi(z))=z_{1}+\left(z_{2}-z_{1}\right)+\cdots+\left(z_{m}-z_{m-1}\right)=z_{m}$, hence the distribution of $L_{F}$ is the same as the distribution of the map

$$
R_{m}: T \ni\left(z_{1}, \ldots, z_{N-1}\right) \mapsto z_{m} .
$$

It follows that

$$
\mathbf{E}_{\mu_{1, n}}\left(L_{F}\right)=\mathbf{E}_{\nu}\left(R_{m}\right)
$$


and the same holds for the variances:

$$
\mathbf{V}_{\mu_{1, n}}\left(L_{F}\right)=\mathbf{V}_{\nu}\left(R_{m}\right)
$$

4. The distribution of $R_{m}$ is well known, its density $h_{m}$ is given by the formula

$$
h_{m}(t)=\frac{(N-1) !}{(m-1) !(N-1-m) !} t^{m-1}(1-t)^{N-1-m}
$$

(see $[1$, p. 64-65]). Therefore,

$$
\begin{aligned}
\mathbf{E}_{\nu}\left(R_{m}\right) & =\int_{0}^{1} t \frac{(N-1) !}{(m-1) !(N-1-m) !} t^{m-1}(1-t)^{N-1-m} d t \\
& =\frac{(N-1) !}{(m-1) !(N-1-m) !} \frac{\Gamma(m+1) \Gamma(N-m)}{\Gamma(N+1)} \\
& =\frac{(N-1) !}{(m-1) !(N-1-m) !} \frac{m !(N-m-1) !}{N !}=\frac{m}{N}, \\
\mathbf{E}_{\nu}\left(R_{m}^{2}\right) & =\int_{0}^{1} t^{2} \frac{(N-1) !}{(m-1) !(N-1-m) !} t^{m-1}(1-t)^{N-1-m} d t \\
& =\frac{(N-1) !}{(m-1) !(N-1-m) !} \frac{\Gamma(m+2) \Gamma(N-m)}{\Gamma(N+2)} \\
& =\frac{(N-1) !}{(m-1) !(N-1-m) !} \frac{(m+1) !(N-m-1) !}{(N+1) !}=\frac{m(m+1)}{N(N+1)},
\end{aligned}
$$

and

$$
\begin{aligned}
\mathbf{V}_{\nu}\left(R_{m}\right) & =\mathbf{E}_{\nu}\left(R_{m}^{2}\right)-\mathbf{E}_{\nu}\left(R_{m}\right)^{2}=\frac{m(m+1)}{N(N+1)}-\frac{m^{2}}{N^{2}} \\
& =\frac{N m(m+1)-(N+1) m^{2}}{N^{2}(N+1)}=\frac{N m^{2}+N m-N m^{2}-m^{2}}{N^{2}(N+1)} \\
& =\frac{m(N-m)}{N^{2}(N+1)} \leqslant \frac{m}{N^{2}} .
\end{aligned}
$$

Coming back to $L_{E_{k}}$, we find that for all $k$ in $\{0, \ldots, n\}$,

$$
\begin{aligned}
& \mathbf{E}_{1, n}(k)=\mathbf{E}_{\mu_{1, n}}\left(L_{E_{k}}\right)=\frac{C_{n}^{k}}{N}=\frac{C_{n}^{k}}{2^{n}}=\mathscr{B}_{n}(k), \\
& \mathbf{V}_{1, n}(k)=\mathbf{V}_{\mu_{1, n}}\left(L_{E_{k}}\right) \leqslant \frac{C_{n}^{k}}{N^{2}}
\end{aligned}
$$

Making use of the Stirling formula, it is easy to see that $C_{n}^{k} \leqslant A \cdot 2^{n} / \sqrt{n}$, where $A$ is a constant independent of $n$. It follows that $\mathbf{V}_{1, n}(k) \leqslant A /\left(2^{n} \sqrt{n}\right)$. Finally, with the Chebyshev inequality we get

$$
\mu_{1, n}\left(\left\{p \in \Delta_{n}:\left|L_{E_{k}}(p)-C_{n}^{k} \cdot 2^{-n}\right| \geqslant \varepsilon\right\}\right) \leqslant \frac{A}{\varepsilon^{2} \cdot 2^{n} \sqrt{n}}
$$


and

$$
\mu_{1, n}\left(\left\{p \in \Delta_{n}: \max _{k=0}^{n}\left|L_{E_{k}}(p)-C_{n}^{k} \cdot 2^{-n}\right| \geqslant \varepsilon\right\}\right) \leqslant(n+1) \frac{A}{\varepsilon^{2} \cdot 2^{n} \sqrt{n}},
$$

therefore

$$
\mu_{1, n}\left(\Delta_{\varepsilon}\right) \geqslant 1-\frac{A \sqrt{n}}{\varepsilon^{2} \cdot 2^{n-1}} .
$$

The second inequality of Theorem 1 follows from the first if we replace $\varepsilon$ by $\varepsilon / n$. Theorem 1 is proved.

If we want to find an explicit value for $A$, we can use the following inequalities instead of the Stirling formula:

$$
\sqrt{2 \pi n} n^{n} \exp \left(-n+\frac{1}{12 n+1}\right) \leqslant n ! \leqslant \sqrt{2 \pi n} n^{n} \exp \left(-n+\frac{1}{12 n}\right)
$$

(see [2, Chap. II, Section 9]). An easy calculation shows that the value $A=2$ works.

5. About the definition of $\mu_{2, n}$. In this section we give two other ways to introduce the measure $\mu_{2, n}$ : Propositions 1 and 2. While Proposition 1 is not needed for the following, Proposition 2 is useful. It replaces the simplices $\Delta_{n}, n \in\{1,2, \ldots\}$ by a single product space endowed with a product probability.

Notation. 1. Denote by $\operatorname{pro}_{n}: \Delta_{n} \rightarrow \Delta_{n-1}$ the map defined by $\operatorname{pro}_{n}\left(\left(p_{i}\right)_{i \in\{0,1\}^{n}}\right)=\left(p_{i}^{\prime}\right)_{i \in\{0,1\}^{n-1}}$, where $p_{i}^{\prime}=p_{(i, 0)}+p_{(i, 1)}$ for all $i \in\{0,1\}^{n-1}$.

2 . For each $i$ in $\{0,1\}^{n}$, denote by $\operatorname{pr}_{i}: \mathbf{R}^{\{0,1\}^{n}} \rightarrow \mathbf{R}$ the map defined by $\operatorname{pr}_{i}\left(\left(p_{j}\right)_{j \in\{0,1\}^{n}}\right)=p_{i}$. It induces a random variable on $\Delta_{n}$ and it is readily seen that for all $i$ in $\{0,1\}^{n-1}, \operatorname{pr}_{(i, 0)}+\operatorname{pr}_{(i, 1)}=\operatorname{pr}_{i} \circ \operatorname{pro}_{n}$ on $\Delta_{n}$.

3 . For an integer $n \geqslant 2$, the map $\psi_{n-1}$ is defined by:

$$
\begin{aligned}
\psi_{n-1}: & \Delta_{n-1} \times[0,1]^{\{0,1\}^{n-1}} \rightarrow \Delta_{n} \\
: & \left(\left(p_{i}\right)_{i \in\{0,1\}^{n-1}},\left(x_{i}\right)_{i \in\{0,1\}^{n-1}}\right) \mapsto\left(p_{(i, j)}^{\prime}\right)_{i \in\{0,1\}^{n-1}, j \in\{0,1\}},
\end{aligned}
$$

where $p_{(i, 0)}^{\prime}=p_{i} x_{i}$ and $p_{(i, 1)}^{\prime}=p_{i}\left(1-x_{i}\right)$.

In the introduction we presented a rather geometric point of view about the probability measures $\mu_{2, n}$. It is possible to give a more probabilistic point of view about these probability measures. The simplex $\Delta_{n}$ is the set of all probability laws of a sequence $\left(X_{1}, \ldots, X_{n}\right)$ of $n$ Bernoulli random variables and the map which associates to each law of $\left(X_{1}, \ldots, X_{n}\right)$ the law of the first $n-1$ Bernoulli random variables $\left(X_{1}, \ldots, X_{n-1}\right)$ is just the projection $\operatorname{pro}_{n}: \Delta_{n} \rightarrow \Delta_{n-1}$. When we know the law of $\left(X_{1}, \ldots, X_{n-1}\right)$ what can we expect about the law of the whole sequence $\left(X_{1}, \ldots, X_{n}\right)$ ? This is given by the conditional distribution given $\operatorname{pro}_{n}$ of the random variables

$$
\frac{\mathbf{P}\left(X_{1}=i_{1}, \ldots, X_{n-1}=i_{n-1}, X_{n}=0\right)}{\mathbf{P}\left(X_{1}=i_{1}, \ldots, X_{n-1}=i_{n-1}\right)}=\frac{\operatorname{pr}_{(i, 0)}}{\operatorname{pr}_{i} \circ \mathrm{pro}_{n}}: \Delta_{n} \rightarrow[0,1],
$$


$i=\left(i_{1}, \ldots, i_{n-1}\right) \in\{0,1\}^{n-1}$. The probability measures $\mu_{2, n}$ are the only ones to ensure that these variables are all uniformly distributed in the interval $[0,1]$ and independent conditionally to the law of $\left(X_{1}, \ldots, X_{n-1}\right)$. This is the meaning of the following proposition which we state without proof.

Proposition 1. The sequence $\left(\mu_{2, n}\right)_{n \geqslant 1}$ is the unique sequence of probability measures such that:

(i) $\mu_{2,1}$ is the normalized Lebesgue measure on $\Delta_{1}$;

(ii) for all integer $n \geqslant 1, \mu_{2, n}$ is a probability on $\Delta_{n}$;

(iii) for all integer $n \geqslant 2$, the image of $\mu_{2, n}$ by $\operatorname{pro}_{n}$ is $\mu_{2, n-1}$;

(iv) for all integer $n \geqslant 2$ and all family $\left(B_{i}\right)_{i \in\{0,1\}^{n-1}}$ of Borel subsets of $[0,1]$,

$$
\mu_{2, n}\left(\frac{\operatorname{pr}_{(i, 0)}}{\operatorname{pr}_{i} \circ \operatorname{pro}_{n}} \in B_{i}, i \in\{0,1\}^{n-1} \mid \operatorname{pro}_{n}\right)=\prod_{i \in\{0,1\}^{n-1}} \lambda\left(B_{i}\right)
$$

where $\lambda$ is the Lebesgue measure on $[0,1]$.

$\mathrm{R}$ e $\mathrm{m}$ a r k. Property (iv) can be replaced as well by: for all integers $n \geqslant 2$ and all family $\left(B_{i}\right)_{i \in\{0,1\}^{n-1}}$ of Borel subsets of $[0,1]$,

$$
\mu_{2, n}\left(\operatorname{pr}_{(i, 0)} \in B_{i}, i \in\{0,1\}^{n-1} \mid \operatorname{pro}_{n}\right)=\prod_{i \in\{0,1\}^{n-1}} \frac{\lambda\left(B_{i} \cap\right] 0, \operatorname{pr}_{i}[)}{\operatorname{pr}_{i}}
$$

where $\lambda$ is the Lebesgue measure on $[0,1]$.

By definition, the probability $\mu_{2, n}$ is the image by $\psi_{n-1}$ of the probability $\mu_{2, n-1} \otimes \lambda_{n-1}$, where $\lambda_{n-1}$ is the Lebesgue measure on $[0,1]^{\{0,1\}^{n-1}}$. We can iterate this process from $n$ down to 1 and we see that the probability measure $\mu_{2, n}$ is the image of the Lebesgue measure on

$$
\Omega_{n}=[0,1]^{\{\varnothing\}} \times[0,1]^{\{0,1\}} \times \cdots \times[0,1]^{\{0,1\}^{n-1}}
$$

by a map $\varphi_{n}: \Omega_{n} \rightarrow \Delta_{n}$. It will be more efficient to define $\varphi_{n}$ on a unique probability space $\Omega$ which does not depend on $n$.

N o t a t i o n. Denote by $\mathscr{J}$ the set $\{\varnothing\} \cup\left(\bigcup_{k=1}^{\infty}\{0,1\}^{k}\right)$ and by $\Omega$ the set $[0,1]^{\mathscr{J}}$.

For $j=\left(j_{1}, \ldots, j_{k}\right)$ in $\mathscr{J}$, denote by $Z_{j}: \Omega=[0,1]^{\mathscr{J}} \rightarrow[0,1]$ the random variable defined by $Z_{j}\left(\left(\omega_{i}\right)_{i \in \mathscr{J}}\right)=\omega_{j}$.

Denote by $Q$ the infinite product of Lebesgue measures on $\Omega$.

For $x$ in $[0,1]$, we put $x^{(0)}=x$ and $x^{(1)}=1-x$.

Proposition 2. For all integers $n \geqslant 1$, consider the map $\varphi_{n}: \Omega \rightarrow$ $\mathbf{R}^{\{0,1\}^{n}}$ defined by

$$
\operatorname{pr}_{\left(i_{1}, \ldots, i_{n}\right)} \circ \varphi_{n}=Z_{\varnothing}^{\left(i_{1}\right)} Z_{\left(i_{1}\right)}^{\left(i_{2}\right)} Z_{\left(i_{1}, i_{2}\right)}^{\left(i_{3}\right)} \cdots Z_{\left(i_{1}, \ldots, i_{n-1}\right)}^{\left(i_{n}\right)} .
$$

Then $\varphi_{n}(\Omega) \subset \Delta_{n}$ and the image by $\varphi_{n}$ of the probability measure $Q$ is $\mu_{2, n}$. 
P r o o f. It is easy to check that $\varphi_{n}(\omega)$ is in $\Delta_{n}$ for all $\omega$ in $\Omega_{n}$. Indeed,

$$
\operatorname{pr}_{\left(i_{1}, \ldots, i_{n}\right)} \circ \varphi_{n}=\left(\operatorname{pr}_{\left(i_{1}, \ldots, i_{n-1}\right)} \circ \varphi_{n-1}\right) Z_{\left(i_{1}, \ldots, i_{n-1}\right)}^{\left(i_{n}\right)},
$$

thus

$$
\begin{aligned}
& \operatorname{pr}_{\left(i_{1}, \ldots, i_{n-1}, 0\right)}\left(\varphi_{n}(\omega)\right)+\operatorname{pr}_{\left(i_{1}, \ldots, i_{n-1}, 1\right)}\left(\varphi_{n}(\omega)\right) \\
& \quad=\operatorname{pr}_{\left(i_{1}, \ldots, i_{n-1}\right)}\left(\varphi_{n-1}(\omega)\right) Z_{\left(i_{1}, \ldots, i_{n-1}\right)}+\operatorname{pr}_{\left(i_{1}, \ldots, i_{n-1}\right)}\left(\varphi_{n-1}(\omega)\right)\left(1-Z_{\left(i_{1}, \ldots, i_{n-1}\right)}\right) \\
& \quad=\operatorname{pr}_{\left(i_{1}, \ldots, i_{n-1}\right)}\left(\varphi_{n-1}(\omega)\right)
\end{aligned}
$$

and it follows by induction that

$$
\begin{aligned}
\sum_{i \in\{0,1\}^{n}} \operatorname{pr}_{i}\left(\varphi_{n}(\omega)\right) & =\sum_{i \in\{0,1\}} \operatorname{pr}_{i}\left(\varphi_{1}(\omega)\right)=\operatorname{pr}_{0}\left(\varphi_{1}(\omega)\right)+\operatorname{pr}_{1}\left(\varphi_{1}(\omega)\right) \\
& =Z_{\varnothing}^{(0)}+Z_{\varnothing}^{(1)}=1
\end{aligned}
$$

Next we prove by induction that the image by $\varphi_{n}$ of the probability measure $Q$ is $\mu_{2, n}$. Suppose the image by $\varphi_{n-1}$ of the probability measure $Q$ is $\mu_{2, n-1}$. Using the sequence of maps $\left(\psi_{n}\right)_{n \geqslant 1}$, it is easy to find a recursion relation satisfied by the sequence of maps $\left(\varphi_{n}\right)_{n \geqslant 1}$ :

$$
\varphi_{n}(\omega)=\psi_{n-1}\left(\varphi_{n-1}(\omega),\left(Z_{i}(\omega)\right)_{i \in\{0,1\}^{n-1}}\right) .
$$

Now $\varphi_{n-1}$ and $\left(Z_{i}\right)_{i \in\{0,1\}^{n-1}}$ are independent random variables, therefore the image by $\Omega \ni \omega \mapsto\left(\varphi_{n-1}(\omega),\left(Z_{i}(\omega)\right)_{i \in\{0,1\}^{n-1}}\right)$ of the probability measure $Q$ is the product of the image by $\varphi_{n-1}$ of $Q$ and of the Lebesgue measure on $[0,1]^{\{0,1\}^{n-1}}$ which is $\lambda_{n-1}$. By induction hypothesis we get $\mu_{2, n-1} \otimes \lambda_{n-1}$ and by definition, the image of $\mu_{2, n-1} \otimes \lambda_{n-1}$ by $\psi_{n-1}$ is $\mu_{2, n}$.

6. Calculation of the first two moments of $\Delta_{n} \ni p \mapsto L_{n}(p)(k)$. Let $n$ be a positive integer. For each subset $F$ of $\{0,1\}^{n}$, we denote by $L_{F}$ the map defined by

$$
\Delta_{n} \ni p=\left(p_{i}\right)_{i \in\{0,1\}^{n}} \mapsto L_{F}(p)=\sum_{i \in F} p_{i} .
$$

For all integers $k$ in $\{0, \ldots, n\}$, we denote by $F_{n, k}$ the subset of elements $i=\left(i_{l}\right)_{l \in\{1, \ldots, n\}} \in\{0,1\}^{n}$ such that $\sum_{l=1}^{n} i_{l}=k$.

Let $n \geqslant 1$ be an integer and let $k$ be an integer in $\{0, \ldots, n\}$. We would like to estimate

$$
\begin{aligned}
\mathbf{E}_{2, n}(k) & =\mathbf{E}_{\mu_{2, n}}\left(L_{n}(\cdot)(k)\right)=\mathbf{E}_{\mu_{2, n}}\left(L_{F_{n, k}}\right) \\
& =\int_{\Delta_{n}} \sum_{i \in F_{n, k}} p_{i} d \mu_{2, n}\left(\left(p_{i}\right)_{i \in\{0,1\}^{n-1}}\right)=\int_{\Omega} \sum_{i \in F_{n, k}} \operatorname{pr}_{i}\left(\varphi_{n}(\omega)\right) d Q(\omega)
\end{aligned}
$$


and

$$
\begin{aligned}
\mathbf{E}_{\mu_{2, n}}\left(L_{n}^{2}(\cdot)(k)\right) & =\mathbf{E}_{\mu_{2, n}}\left(L_{F_{n, k}}^{2}\right)=\int_{\Delta_{n}}\left(\sum_{i \in F_{n, k}} p_{i}\right)^{2} d \mu_{2, n}\left(\left(p_{i}\right)_{i \in\{0,1\}^{n-1}}\right) \\
& =\int_{\Omega}\left(\sum_{i \in F_{n, k}} \operatorname{pr}_{i}\left(\varphi_{n}(\omega)\right)\right)^{2} d Q(\omega) .
\end{aligned}
$$

Set $f_{n, k}=\sum_{i \in F_{n, k}} \operatorname{pr}_{i} \circ \varphi_{n}$.

1. By Proposition 2,

$$
\begin{aligned}
\mathbf{E}_{Q}\left(f_{n, k}\right) & =\mathbf{E}_{Q}\left(\sum_{i \in F_{n, k}} \operatorname{pr}_{i} \circ \varphi_{n}\right) \\
& =\mathbf{E}_{Q}\left(\sum_{\left(i_{1}, \ldots, i_{n}\right) \in F_{n, k}}\left(\operatorname{pr}_{\left(i_{1}, \ldots, i_{n-1}\right)} \circ \varphi_{n-1}\right) Z_{\left(i_{1}, \ldots, i_{n-1}\right)}^{\left(i_{n}\right)}\right) \\
& =\sum_{\left(i_{1}, \ldots, i_{n}\right) \in F_{n, k}} \mathbf{E}_{Q}\left(\left(\operatorname{pr}_{\left(i_{1}, \ldots, i_{n-1}\right)} \circ \varphi_{n-1}\right) Z_{\left(i_{1}, \ldots, i_{n-1}\right)}^{\left(i_{n}\right)}\right),
\end{aligned}
$$

and since $\varphi_{n-1}$ and $Z_{\left(i_{1}, \ldots, i_{n-1}\right)}^{\left(i_{n}\right)}$ are independent, we get

$$
\begin{aligned}
\mathbf{E}_{Q}\left(f_{n, k}\right) & =\sum_{\left(i_{1}, \ldots, i_{n}\right) \in F_{n, k}} \mathbf{E}_{Q}\left(\operatorname{pr}_{\left(i_{1}, \ldots, i_{n-1}\right)} \circ \varphi_{n-1}\right) \mathbf{E}_{Q}\left(Z_{\left(i_{1}, \ldots, i_{n-1}\right)}^{\left(i_{i_{n}}\right)}\right) \\
& =\frac{1}{2} \sum_{\left(i_{1}, \ldots, i_{n}\right) \in F_{n, k}} \mathbf{E}_{Q}\left(\operatorname{pr}_{\left(i_{1}, \ldots, i_{n-1}\right)} \circ \varphi_{n-1}\right) .
\end{aligned}
$$

Furthermore, $F_{n, k}=F_{n-1, k-1} \times\{1\} \cup F_{n-1, k} \times\{0\}$, thus

$$
\begin{aligned}
\mathbf{E}_{Q}\left(f_{n, k}\right)= & \frac{1}{2}\left(\sum_{\left(i_{1}, \ldots, i_{n}\right) \in F_{n-1, k-1} \times\{1\}} \mathbf{E}_{Q}\left(\operatorname{pr}_{\left(i_{1}, \ldots, i_{n-1}\right)} \circ \varphi_{n-1}\right)\right. \\
& \left.+\sum_{\left(i_{1}, \ldots, i_{n}\right) \in F_{n-1, k} \times\{0\}} \mathbf{E}_{Q}\left(\operatorname{pr}_{\left(i_{1}, \ldots, i_{n-1}\right)} \circ \varphi_{n-1}\right)\right) \\
= & \frac{1}{2}\left(\mathbf{E}_{Q}\left(f_{n-1, k-1}\right)+\mathbf{E}_{Q}\left(f_{n-1, k}\right)\right) .
\end{aligned}
$$

We have also $\mathbf{E}_{Q}\left(f_{1,0}\right)=\mathbf{E}_{Q}\left(Z_{\varnothing}^{(0)}\right)=\mathbf{E}_{Q}\left(Z_{\varnothing}^{(1)}\right)=\mathbf{E}_{Q}\left(f_{1,1}\right)=1 / 2$, therefore, by induction, we get $\mathbf{E}_{Q}\left(f_{n, k}\right)=C_{n}^{k} \cdot 2^{-n}$. Hence

$$
\mathbf{E}_{2, n}(k)=C_{n}^{k} \cdot 2^{-n} .
$$

2. The quadratic mean $\mathbf{E}_{Q}\left(f_{n, k}^{2}\right)$ is a little more difficult to estimate. The main idea is to decompose $F_{n, k}$ into two sets

$$
F_{n, k}^{0}=\left\{\left(i_{1}, \ldots, i_{n}\right) \in F_{n, k}: i_{1}=0\right\}, \quad F_{n, k}^{1}=\left\{\left(i_{1}, \ldots, i_{n}\right) \in F_{n, k}: i_{1}=1\right\}
$$


and to observe that for each $i$ in $F_{n, k}^{0}$ and each $j$ in $F_{n, k}^{1}$, the two variables

$$
Z_{\left(i_{1}\right)}^{\left(i_{2}\right)} Z_{\left(i_{1}, i_{2}\right)}^{\left(i_{3}\right)} \cdots Z_{\left(i_{1}, \ldots, i_{n-1}\right)}^{\left(i_{n}\right)}, \quad Z_{\left(j_{1}\right)}^{\left(j_{2}\right)} Z_{\left(j_{1}, j_{2}\right)}^{\left(j_{3}\right)} \cdots Z_{\left(j_{1}, \ldots, j_{n-1}\right)}^{\left(j_{n}\right)}
$$

are independent. We have

$$
\begin{aligned}
\mathbf{E}_{Q}\left(f_{n, k}^{2}\right)= & \mathbf{E}_{Q}\left(\left(\sum_{i \in F_{n, k}^{0}} \operatorname{pr}_{i} \circ \varphi_{n}\right)^{2}\right)+\mathbf{E}_{Q}\left(\left(\sum_{i \in F_{n, k}^{1}} \operatorname{pr}_{i} \circ \varphi_{n}\right)^{2}\right) \\
& +2 \mathbf{E}_{Q}\left(\left(\sum_{i \in F_{n, k}^{0}} \operatorname{pr}_{i} \circ \varphi_{n}\right)\left(\sum_{j \in F_{n, k}^{1}} \operatorname{pr}_{j} \circ \varphi_{n}\right)\right)=T_{1}+T_{2}+2 T_{3} .
\end{aligned}
$$

The first term gives

$$
\begin{aligned}
T_{1} & =\mathbf{E}_{Q}\left(\left(\sum_{i \in F_{n, k}^{0}} Z_{\varnothing}^{(0)} Z_{(0)}^{\left(i_{2}\right)} Z_{\left(0, i_{2}\right)}^{\left(i_{3}\right)} \cdots Z_{\left(0, i_{2}, \ldots, i_{n-1}\right)}^{\left(i_{n}\right)}\right)^{2}\right) \\
& =\mathbf{E}_{Q}\left(Z_{\varnothing}^{2}\left(\sum_{i \in F_{n, k}^{0}} Z_{(0)}^{\left(i_{2}\right)} Z_{\left(0, i_{2}\right)}^{\left(i_{3}\right)} \cdots Z_{\left(0, i_{2}, \ldots, i_{n-1}\right)}^{\left(i_{n}\right)}\right)^{2}\right) .
\end{aligned}
$$

Since $Z_{\varnothing}$ is independent of the other $Z_{i}$, we get

$$
\begin{aligned}
\mathbf{E}_{Q} & \left(\left(\sum_{i \in F_{n, k}^{0}} \operatorname{pr}_{i} \circ \varphi_{n}\right)^{2}\right) \\
& =\mathbf{E}_{Q}\left(Z_{\varnothing}^{2}\right) \mathbf{E}_{Q}\left(\left(\sum_{i \in F_{n, k}^{0}} Z_{(0)}^{\left(i_{2}\right)} Z_{\left(0, i_{2}\right)}^{\left(i_{3}\right)} \cdots Z_{\left(0, i_{2}, \ldots, i_{n-1}\right)}^{\left(i_{n}\right)}\right)^{2}\right) .
\end{aligned}
$$

The last thing to see for the computation of $T_{1}$ is that

$$
\begin{aligned}
& \mathbf{E}_{Q}\left(\left(\sum_{i \in F_{n, k}^{0}} Z_{(0)}^{\left(i_{2}\right)} Z_{\left(0, i_{2}\right)}^{\left(i_{3}\right)} \cdots Z_{\left(0, i_{2}, \ldots, i_{n-1}\right)}^{\left(i_{n}\right)}\right)^{2}\right) \\
& \quad=\mathbf{E}_{Q}\left(\left(\sum_{i \in F_{n-1, k}} Z_{(\varnothing)}^{\left(i_{1}\right)} Z_{\left(i_{1}\right)}^{\left(i_{2}\right)} \cdots Z_{\left(i_{1}, \ldots, i_{n-2}\right)}^{\left(i_{n-1}\right)}\right)^{2}\right)=\mathbf{E}_{Q}\left(f_{n-1, k}^{2}\right),
\end{aligned}
$$

thus

$$
T_{1}=\frac{1}{3} \mathbf{E}_{Q}\left(f_{n-1, k}^{2}\right)
$$

Exactly the same arguments show that

$$
T_{2}=\frac{1}{3} \mathbf{E}_{Q}\left(f_{n-1, k-1}^{2}\right) .
$$


By independence, the last term gives

$$
\begin{aligned}
T_{3}= & \mathbf{E}_{Q}\left(\left(\sum_{i \in F_{n, k}^{0}} Z_{\varnothing}^{(0)} Z_{(0)}^{\left(i_{2}\right)} Z_{\left(0, i_{2}\right)}^{\left(i_{3}\right)} \cdots Z_{\left(0, i_{2}, \ldots, i_{n-1}\right)}^{\left(i_{n}\right)}\right)\right. \\
& \left.\times\left(\sum_{i \in F_{n, k}^{1}} Z_{\varnothing}^{(1)} Z_{(1)}^{\left(i_{2}\right)} Z_{\left(1, i_{2}\right)}^{\left(i_{3}\right)} \cdots Z_{\left(1, i_{2}, \ldots, i_{n-1}\right)}^{\left(i_{n}\right)}\right)\right) \\
= & \mathbf{E}_{Q}\left(Z_{\varnothing}^{(0)} Z_{\varnothing}^{(1)}\right) \mathbf{E}_{Q}\left(\sum_{i \in F_{n, k}^{0}} Z_{(0)}^{\left(i_{2}\right)} Z_{\left(0, i_{2}\right)}^{\left(i_{3}\right)} \cdots Z_{\left(0, i_{2}, \ldots, i_{n-1}\right)}^{\left(i_{n}\right)}\right) \\
& \times \mathbf{E}_{Q}\left(\sum_{i \in F_{n, k}^{1}} Z_{(1)}^{\left(i_{2}\right)} Z_{\left(1, i_{2}\right)}^{\left(i_{3}\right)} \cdots Z_{\left(0, i_{2}, \ldots, i_{n-1}\right)}^{\left(i_{n}\right)}\right)=\frac{1}{6} \mathbf{E}_{Q}\left(f_{n-1, k}\right) \mathbf{E}_{Q}\left(f_{n-1, k-1}\right) .
\end{aligned}
$$

Finally, we get the relation

$$
\mathbf{E}_{Q}\left(f_{n, k}^{2}\right)=\frac{1}{3}\left[\mathbf{E}_{Q}\left(f_{n-1, k}^{2}\right)+\mathbf{E}_{Q}\left(f_{n-1, k-1}^{2}\right)+\mathbf{E}_{Q}\left(f_{n-1, k}\right) \mathbf{E}_{Q}\left(f_{n-1, k-1}\right)\right] .
$$

3. The recursion relation (1) and the equality $\mathbf{E}_{Q}\left(f_{m, l}\right)=2^{-m} C_{m}^{l}$ enable us to find a recursion relation between $\mathbf{V}_{Q}\left(f_{n-1, k-1}\right), \mathbf{V}_{Q}\left(f_{n-1, k}\right)$, and $\mathbf{V}_{Q}\left(f_{n, k}\right)$ :

$$
\begin{aligned}
\mathbf{V}_{Q}\left(f_{n, k}\right)= & \mathbf{E}_{Q}\left(f_{n, k}^{2}\right)-\mathbf{E}_{Q}\left(f_{n, k}\right)^{2} \\
= & \frac{1}{3}\left[\mathbf{E}_{Q}\left(f_{n-1, k}^{2}\right)+\mathbf{E}_{Q}\left(f_{n-1, k-1}^{2}\right)+\mathbf{E}_{Q}\left(f_{n-1, k}\right) \mathbf{E}_{Q}\left(f_{n-1, k-1}\right)\right] \\
& -\frac{1}{4}\left[\mathbf{E}_{Q}\left(f_{n-1, k}\right)+\mathbf{E}_{Q}\left(f_{n-1, k-1}\right)\right]^{2} \\
= & \frac{1}{3}\left(\mathbf{V}_{Q}\left(f_{n-1, k}\right)+\mathbf{V}_{Q}\left(f_{n-1, k-1}\right)+\mathbf{E}_{Q}\left(f_{n-1, k}\right) \mathbf{E}_{Q}\left(f_{n-1, k-1}\right)\right) \\
& +\frac{1}{12}\left[\mathbf{E}_{Q}\left(f_{n-1, k}\right)^{2}+\mathbf{E}_{Q}\left(f_{n-1, k-1}\right)^{2}\right]-\frac{1}{2} \mathbf{E}_{Q}\left(f_{n-1, k}\right) \mathbf{E}_{Q}\left(f_{n-1, k-1}\right) \\
= & \frac{1}{3}\left(\mathbf{V}_{Q}\left(f_{n-1, k}\right)+\mathbf{V}_{Q}\left(f_{n-1, k-1}\right)\right)+\frac{1}{12}\left(\mathbf{E}_{Q}\left(f_{n-1, k}\right)-\mathbf{E}_{Q}\left(f_{n-1, k-1}\right)\right)^{2} \\
= & \frac{1}{3}\left(\mathbf{V}_{Q}\left(f_{n-1, k}\right)+\mathbf{V}_{Q}\left(f_{n-1, k-1}\right)\right)+\frac{1}{12}\left[2^{-n+1}\left(C_{n-1}^{k}-C_{n-1}^{k-1}\right)\right]^{2} .
\end{aligned}
$$

Hence,

$$
\begin{aligned}
\mathbf{V}_{\mu_{2, n}}\left(L_{F_{n, k}}\right)= & \frac{1}{3}\left[\mathbf{V}_{\mu_{2, n-1}}\left(L_{F_{n-1, k}}\right)+\mathbf{V}_{\mu_{2, n-1}}\left(L_{F_{n-1, k-1}}\right)\right] \\
& +\frac{1}{12}\left[2^{-n+1}\left(C_{n-1}^{k-1}-C_{n-1}^{k}\right)\right]^{2}
\end{aligned}
$$

6.1. An upper bound for $\mathbf{V}_{2, n}(k)=\mathbf{V}_{\mu_{2, n}}\left(L_{F_{n, k}}\right)$. We need the following lemma. 
Lemma 1. There exists a constant $C$ such that for all integers $n \geqslant 1$ we have:

1) for all $k$ in $\{0, \ldots, n\}$,

$$
2^{-n}\left|C_{n}^{k-1}-C_{n}^{k}\right| \leqslant \frac{C}{n}
$$

2) for all $k$ in $\{0, \ldots, n\}$ such that $|k-n / 2| \geqslant \sqrt{n \ln n}$,

$$
2^{-n}\left|C_{n}^{k-1}-C_{n}^{k}\right| \leqslant \frac{C}{n^{5 / 2}} .
$$

P r o o f. In what follows, $C$ denotes a constant whose value may change at each line. Since

$$
\left.2^{-n}\left|C_{n}^{k-1}-C_{n}^{k}\right|=2^{-n} \frac{n !}{(n-k) ! k !} \mid \frac{k}{n-k+1}-1\right]\left|=2^{-n} C_{n}^{k}\right| \frac{n+1-2 k}{n+1-k} \mid,
$$

we can use the classical Laplace-Moivre estimate about the binomial law: Let $\left(a_{n}\right)_{n \geqslant 1}$ be a sequence of nonnegative real numbers which go to 0 as $n$ goes to infinity. Then for all positive integers $n$ and all integers $k$ such that $|k-n / 2| \leqslant a_{n} n^{2 / 3}$ we have

$$
2^{-n} C_{n}^{k}=\frac{1+\delta_{n}(k)}{\sqrt{\pi n / 2}} \exp \left(-\frac{2(k-n / 2)^{2}}{n}\right)
$$

where

$$
\lim _{n \rightarrow \infty} \sup _{k:|k-n / 2| \leqslant a_{n} n^{2 / 3}}\left|\delta_{n}(k)\right|=0
$$

(actually, it is a slight extension of the Laplace-Moivre theorem which deals only with integers $k$ such that $|k-n / 2| \leqslant a \sqrt{n}$, where $a$ is a fixed real number; see [2, Chap. VII, Section 3, Theorem 1] or [3, p. 36, Proposition 8.2]). It follows that for all positive integers $n$ and all integers $k$ such that $|k-n / 2| \leqslant$ $a_{n} n^{2 / 3}$,

$$
2^{-n} C_{n}^{k} \leqslant \frac{C}{\sqrt{n}} \exp \left(-\frac{2(k-n / 2)^{2}}{n}\right),
$$

where the constant $C$ does not depend on $n$. Making use of the monotonicity of the binomial coefficients, we get the following inequality:

$$
2^{-n} C_{n}^{k} \leqslant \frac{C}{n^{5 / 2}}
$$

for all integers $n$ and all integers $k$ such that $|k-n / 2| \geqslant \sqrt{n \ln n}$. This last inequality implies assertion 2). Now let us prove assertion 1). For all positive integers $n$ and all integers $k$ such that $|k-n / 2| \leqslant \sqrt{n \ln n}$, we have

$$
2^{-n}\left|C_{n}^{k-1}-C_{n}^{k}\right| \leqslant \frac{C}{\sqrt{n}} \exp \left(-\frac{2(k-n / 2)^{2}}{n}\right) \frac{|n-2 k|+1}{n} .
$$


Put $t=(k-n / 2) / \sqrt{n}$. We get

$$
2^{-n}\left|C_{n}^{k-1}-C_{n}^{k}\right| \leqslant \frac{C|t|}{n} \exp \left(-2 t^{2}\right)+\frac{C}{n^{3 / 2}}
$$

and since the function $\mathbf{R} \ni t \mapsto|t| e^{-2 t^{2}}$ is bounded, $2^{-n}\left|C_{n}^{k-1}-C_{n}^{k}\right| \leqslant C / n$. Lemma 1 is proved.

Proposition 3. There exists a constant $C$ such that for all positive integers $n$,

$$
v_{n}:=\sup _{k \in\{0, \ldots, n\}} \mathbf{V}_{\mu_{2, n}}\left(L_{F_{n, k}}\right) \leqslant \frac{C}{n^{2}} .
$$

P r o o f. Let $n \geqslant 2$ be an integer. Since for all $k$ in $\{0, \ldots, n\}$,

$$
\begin{aligned}
\mathbf{V}_{\mu_{2, n}}\left(L_{F_{n, k}}\right)= & \frac{1}{3}\left(\mathbf{V}_{\mu_{2, n-1}}\left(L_{F_{n-1, k-1}}\right)+\mathbf{V}_{\mu_{2, n-1}}\left(L_{F_{n-1, k}}\right)\right) \\
& +\frac{1}{12}\left[2^{-n+1}\left(C_{n-1}^{k-1}-C_{n-1}^{k}\right)\right]^{2}
\end{aligned}
$$

we have

$$
v_{n} \leqslant \frac{2}{3} v_{n-1}+\frac{1}{12}\left[2^{-n+1}\left(C_{n-1}^{k-1}-C_{n-1}^{k}\right)\right]^{2} .
$$

Lemma 1 implies that $v_{n} \leqslant(2 / 3) v_{n-1}+C / n^{2}$. By induction, we get that for all integers $n \geqslant 2$,

$$
v_{n} \leqslant\left(\frac{2}{3}\right)^{n-1} v_{1}+C \sum_{i=0}^{n-2}(n-i)^{-2}\left(\frac{2}{3}\right)^{i}
$$

The sum $\sum_{i=0}^{n-2}(n-i)^{-2}(2 / 3)^{i}$ is easy to estimate by dividing it into two sums, $\sum_{0 \leqslant i \leqslant n / 2}$ and $\sum_{n / 2<i \leqslant n-2}$ : since

$$
\begin{aligned}
& \sum_{0 \leqslant i \leqslant n / 2} \frac{1}{(n-i)^{2}}\left(\frac{2}{3}\right)^{i} \leqslant \frac{4}{n^{2}} \sum_{0 \leqslant i \leqslant n / 2}\left(\frac{2}{3}\right)^{i} \leqslant \frac{12}{n^{2}}, \\
& \sum_{n / 2<i \leqslant n-2} \frac{1}{(n-i)^{2}}\left(\frac{2}{3}\right)^{i} \leqslant \frac{n}{2}\left(\frac{2}{3}\right)^{n / 2} \leqslant \frac{C}{n^{2}},
\end{aligned}
$$

we have

$$
v_{n} \leqslant\left(\frac{2}{3}\right)^{n} v_{1}+\frac{C}{n^{2}} \leqslant \frac{C}{n^{2}} .
$$

Proposition 3 is proved.

Proposition 4. There exists a constant $C$ such that for all positive integers $n$

$$
u_{n}:=\sup _{k \in\{0, \ldots, n\}:|k-n / 2| \geqslant 2 \sqrt{n \ln n}} \mathbf{V}_{\mu_{2, n}}\left(L_{F_{n, k}}\right) \leqslant \frac{C}{n^{5}}
$$


P r o of. Let $n \geqslant 2$ be an integer. First note that the two variables $L_{F_{n, k}}$ and $L_{F_{n, n-k}}$ have the same law, so it suffices to prove the proposition for $k \geqslant n / 2$.

By Lemma 1 , for all $k$ in $\{0, \ldots, n\}$ such that $k-n / 2 \geqslant \sqrt{n \ln n}$, we have

$$
\begin{aligned}
\mathbf{V}_{\mu_{2, n}}\left(L_{F_{n, k}}\right)= & \frac{1}{3}\left[\mathbf{V}_{\mu_{2, n-1}}\left(L_{F_{n-1, k-1}}\right)+\mathbf{V}_{\mu_{2, n-1}}\left(L_{F_{n-1, k}}\right)\right] \\
& +\frac{1}{12}\left[2^{-n+1}\left(C_{n-1}^{k-1}-C_{n-1}^{k}\right)\right]^{2} \\
\leqslant & \frac{1}{3}\left[\mathbf{V}_{\mu_{2, n-1}}\left(L_{F_{n-1, k-1}}\right)+\mathbf{V}_{\mu_{2, n-1}}\left(L_{F_{n-1, k}}\right)\right]+\frac{C}{n^{5}} .
\end{aligned}
$$

Fix an integer $k \geqslant n / 2+2 \sqrt{n \ln n}$. We prove by induction on $l$ that for all integer $l \leqslant \sqrt{n \ln n}$,

$$
\mathbf{V}_{\mu_{2, n}}\left(L_{F_{n, k}}\right) \leqslant \frac{1}{3^{l}} \sum_{i=0}^{l} C_{l}^{i} \mathbf{V}_{\mu_{2, n-l}}\left(L_{F_{n-l, k-i}}\right)+C \sum_{i=0}^{l-1} \frac{1}{(n-i)^{5}}\left(\frac{2}{3}\right)^{i} .
$$

Indeed, if $l \leqslant \sqrt{n \ln n}$, then for all $i \in\{0, \ldots, l\}$,

$$
k-i-\frac{n-l}{2} \geqslant \sqrt{n \ln n} \geqslant \sqrt{(n-l) \ln (n-l)},
$$

therefore

$$
\begin{aligned}
\mathbf{V}_{\mu_{2, n-l}}\left(L_{F_{n-l, k-i}}\right) \leqslant & \frac{1}{3}\left(\mathbf{V}_{\mu_{2, n-l-1}}\left(L_{F_{n-l-1, k-i-1}}\right)+\mathbf{V}_{\mu_{2, n-l-1}}\left(L_{F_{n-l-1, k-i}}\right)\right) \\
& +\frac{C}{(n-l)^{5}} .
\end{aligned}
$$

Together with the induction hypothesis, this imply that

$$
\begin{aligned}
\mathbf{V}_{\mu_{2, n}}\left(L_{F_{n, k}}\right) \leqslant & \frac{1}{3^{l}} \sum_{i=0}^{l} C_{l}^{i}\left\{\frac{1}{3}\left[\mathbf{V}_{\mu_{2, n-l-1}}\left(L_{F_{n-l-1, k-i-1}}\right)+\mathbf{V}_{\mu_{2, n-l-1}}\left(L_{F_{n-l-1, k-i}}\right)\right]\right. \\
& \left.\quad+\frac{C}{(n-l)^{5}}\right\}+C \sum_{i=0}^{l-1} \frac{1}{(n-i)^{5}}\left(\frac{2}{3}\right)^{i} \\
= & \frac{1}{3^{l+1}}\left\{\left[\mathbf{V}_{\mu_{2, n-(l+1)}}\left(L_{F_{n-(l+1), k-(l+1)}}\right)+\mathbf{V}_{\mu_{2, n-l}(l+1)}\left(L_{F_{n-l-1, k}}\right)\right]\right. \\
& \left.+\sum_{j=1}^{l}\left(C_{l}^{j-1}+C_{l}^{j}\right) \mathbf{V}_{\mu_{2, n-(l+1)}}\left(L_{F_{n-(l+1), k-j}}\right)\right\} \\
& +C \sum_{j=0}^{l} \frac{1}{(n-j)^{5}}\left(\frac{2}{3}\right)^{j} \\
= & \frac{1}{3^{l+1}} \sum_{i=0}^{l+1} C_{l+1}^{i} \mathbf{V}_{\mu_{2, n-(l+1)}}\left(L_{F_{n-(l+1), k-i}}\right)+C \sum_{j=0}^{l} \frac{1}{(n-j)^{5}}\left(\frac{2}{3}\right)^{j}
\end{aligned}
$$


As before, it is easy to prove that $\sum_{j=0}^{l-1}(n-j)^{-5}(2 / 3)^{j} \leqslant C / n^{5}$. Furthermore (recall that $0 \leqslant L_{F} \leqslant 1$ ),

$$
\frac{1}{3^{l}} \sum_{i=0}^{l} C_{l}^{i} \mathbf{V}_{\mu_{2, n-l}}\left(L_{F_{n-l, k-i}}\right) \leqslant \frac{1}{3^{l}} \sum_{i=0}^{l} C_{l}^{i}=\left(\frac{2}{3}\right)^{l} .
$$

Thus, with $l=\sqrt{n \ln n}$ we find that

$$
\begin{aligned}
\mathbf{V}_{\mu_{2, n}}\left(L_{F_{n, k}}\right) & \leqslant \frac{1}{3^{l}} \sum_{i=0}^{l} C_{l}^{i} \mathbf{V}_{\mu_{2, n-l}}\left(L_{F_{n-l, k-i}}\right)+C \sum_{i=0}^{l-1} \frac{1}{(n-i)^{5}}\left(\frac{2}{3}\right)^{i} \\
& \leqslant\left(\frac{2}{3}\right)^{\sqrt{n \ln n}}+\frac{C}{n^{5}} \leqslant \frac{C}{n^{5}} .
\end{aligned}
$$

Proposition 4 is proved.

6.2. End of the proof of Theorem 2. Let $k$ be an integer between $n / 2-2 \sqrt{n \ln n}$ and $n / 2+2 \sqrt{n \ln n}$. By Proposition 3 and the Chebyshev inequality, for all positive $\varepsilon$,

$$
\mu_{2, n}\left(\left\{p \in \Delta_{n}:\left|L_{F_{n, k}}(p)-C_{n}^{k} \cdot 2^{-n}\right| \geqslant \varepsilon\right\}\right) \leqslant \frac{C}{\varepsilon^{2} n^{2}},
$$

thus

$$
\mu_{2, n}\left(\left\{p \in \Delta_{n}: \max ^{*}\left|L_{F_{n, k}}(p)-C_{n}^{k} \cdot 2^{-n}\right| \geqslant \varepsilon\right\}\right) \leqslant 4 \sqrt{n \ln n} \frac{C}{\varepsilon^{2} n^{2}},
$$

where $\max ^{*}$ is taken over $n / 2-2 \sqrt{n \ln n} \leqslant k \leqslant n / 2+2 \sqrt{n \ln n}$. Let $k$ be an integer in $\{0, \ldots, n\}$ such that $|n / 2-k| \geqslant 2 \sqrt{n \ln n}$. By Proposition 4 and the Chebyshev inequality, for all positive numbers $\varepsilon$,

$$
\mu_{2, n}\left(\left\{p \in \Delta_{n}:\left|L_{F_{n, k}}(p)-C_{n}^{k} \cdot 2^{-n}\right| \geqslant \varepsilon\right\}\right) \leqslant \frac{C}{\varepsilon^{2} n^{5}},
$$

thus

$$
\mu_{2, n}\left(\left\{p \in \Delta_{n}: \max _{k:|n / 2-k| \geqslant 2 \sqrt{n \ln n}}\left|L_{F_{n, k}}(p)-C_{n}^{k} \cdot 2^{-n}\right| \geqslant \varepsilon\right\}\right) \leqslant n \frac{C}{\varepsilon^{2} n^{5}} .
$$

It follows that

$$
\mu_{2, n}\left(\left\{p \in \Delta_{n}: \max _{k \in\{0, \ldots, n\}}\left|L_{n}(p)(k)-L_{n}\left(\mathscr{B}_{n}\right)(p)\right| \leqslant \varepsilon\right\}\right) \geqslant 1-\frac{C \ln n}{\varepsilon^{2} n^{3 / 2}} .
$$

Let $\delta$ be a positive number. We shall use inequality (2) with $\varepsilon_{1}=$ $\delta /(4 \sqrt{n \ln n})$ and inequality (3) with $\varepsilon_{2}=\delta / n$. For each subset $I$ of $\{0, \ldots, n\}$, the set

$$
\left\{p \in \Delta_{n}:\left|L_{n}(p)(I)-L_{n}\left(\mathscr{B}_{n}\right)(I)\right| \geqslant \delta\right\}
$$


is included in the union of

$$
\bigcup_{k:|n / 2-k| \leqslant 2 \sqrt{n \ln n}}\left\{p \in \Delta_{n}:\left|L_{n}(p)(k)-L_{n}\left(\mathscr{B}_{n}\right)(k)\right| \geqslant \frac{\delta}{4 \sqrt{n \ln n}}\right\}
$$

and

$$
\bigcup_{k:|n / 2-k| \geqslant 2 \sqrt{n \ln n}}\left\{p \in \Delta_{n}:\left|L_{n}(p)(k)-L_{n}\left(\mathscr{B}_{n}\right)(k)\right| \geqslant \frac{\delta}{n}\right\}
$$

which does not depend on $I$, therefore (recall that $L_{n}(p)(k)=L_{F_{n, k}}(p)$ )

$$
\begin{aligned}
& \mu_{2, n}\left(\left\{p \in \Delta_{n}: \sup _{I \subset\{1, \ldots, n\}}\left|L_{n}(p)(I)-L_{n}\left(\mathscr{B}_{n}\right)(I)\right| \geqslant \delta\right)\right\} \\
& \leqslant \sum_{k:|n / 2-k| \leqslant 2 \sqrt{n \ln n}} \mu_{2, n}\left(\left\{p \in \Delta_{n}:\left|L_{n}(p)(k)-L_{n}\left(\mathscr{B}_{n}\right)(k)\right| \geqslant \frac{\delta}{4 \sqrt{n \ln n}}\right\}\right) \\
& \quad+\sum_{k:|n / 2-k| \geqslant 2 \sqrt{n \ln n}} \mu_{2, n}\left(\left\{p \in \Delta_{n}:\left|L_{n}(p)(k)-L_{n}\left(\mathscr{B}_{n}\right)(k)\right| \geqslant \frac{\delta}{n}\right\}\right) \\
& \leqslant \frac{C \sqrt{n \ln n}}{\varepsilon_{1}^{2} n^{2}}+\frac{C}{\varepsilon_{2}^{2} n^{4}}=\frac{C}{\delta^{2}}\left(\frac{16 \sqrt{n} \ln ^{3 / 2} n}{n}+\frac{1}{n^{2}}\right) \\
& \leqslant \frac{C \ln ^{3 / 2} n}{\sqrt{n} \delta^{2}} .
\end{aligned}
$$

Theorem 2 is proved.

\section{REFERENCES}

1. Dacunha-Castelle D., Duflo $M$. Exercices de probabilités et statistiques. 1. Problèmes à temps fixe. Paris: Masson, 1982.

2. Феллер В. Введение в теорию вероятностей и ее приложения, т. 1. М.: Мир, 1984, $527 \mathrm{c}$.

3. Lesigne E. Pile ou face: une introduction au calcul des probabilités. Paris: Ellipse, 2001, $117 \mathrm{p}$.

4. Takeuchi K., Takemura A. On sum of 0-1 random variables. I: Univariate case. - Ann. Inst. Statist. Math., 1987, v. 39, № 1, p. 85-102.

Поступила в редакцию

9.IX.2009

Исправленный вариант 30.X.2009 